\title{
THE FREENESS OF A GROUP BASED ON A DISTRIBUTIVE LATTICE ${ }^{1}$
}

\author{
P. HILL AND H. SUBRAMANIAN
}

\begin{abstract}
Let $L$ be a distributive lattice and $G$ the abelian group with the following presentation. The generators of $G$ are the elements of the lattice $L$, and the relations are $(a \vee b)+(a \wedge b)=$ $a+b$ where $a$ and $b$ are arbitrary elements of $L$. It is shown that $G$ is free abelian. In particular, $G$ is torsion free. The latter statement answers affirmatively a question posed several years ago by E. Weinberg.
\end{abstract}

This brief note is in the nature of an addendum to an earlier paper by one of the authors [2]. Our purpose is to prove that an abelian group based on a distributive lattice remains free under the relations $(a \vee b)+(a \wedge b)$ $=a+b$. This result is basically a corollary of the two theorems in [2], but the application was previously overlooked. In fact, the second-named author initially proved our result, without reference to [2], using a theorem of G. Nöbeling [4]. However, it is the more recent results of [1] and [2] that yield the short proof that follows.

Theorem. Let $L$ be an arbitrary distributive lattice and let $G$ be the abelian group generated by the elements of $L$ with relations $(a \vee b)+$ $(a \wedge b)=a+b$ for all $a, b \in L$. Then $G$ is free.

Proof. Denote by $F$ the free abelian group based on the distributive lattice $L$ (with no relations at all on $F$ ). Then $F$ is isomorphic to the additive group of the semigroup ring $Z[S]$ where $S$ is the semigroup associated with the set $L$ and the operation $\wedge$ alone. The advantage of this identification is that $F$ is now endowed, in a natural way, with multiplication and a ring structure. Indeed, $F$ is a commutative ring generated by idempotents. We shall denote the additive structure of $F$ by $\mathrm{F}^{+}$and, more generally, follow the notation of [2].

Received by the editors June 10, 1974 .

AMS (MOS) subject classifications (1970). Primary 20K05, 20K15, 20K20, 20K25; Secondary 16A32, 06A35.

Key words and phrases. Distributive lattice, semigroup ring, free abelian group, idempotent generators, pure subgroup.

1 This research was supported in part by NSF grant GP-38071X. 
Let $R$ be the subgroup of $F^{+}$generated by the elements $(x \vee y)+$ $(x \wedge y)-x-y$ with $x$ and $y$ arbitrary elements of $L$. Using the distributive property of $L$, we observe that $z(x \vee y)=(z \wedge x) \vee(z \wedge y)$. Consequently, the equation

$$
\begin{aligned}
z[(x \vee y)+(x \wedge y)-x-y] & \\
= & (z \wedge x) \vee(z \wedge y)+(z \wedge x) \wedge(z \wedge y)_{-}(z \wedge x)-(z \wedge y)
\end{aligned}
$$

is valid in the semigroup ring $F=Z[S]$ for all $x, y$ and $z$ in $L$, which implies that $R$ is an ideal of $F$. Therefore, if $G$ denotes $F^{+} / R^{+}=(F / R)^{+}$, Theorem 2 in [2] implies that $G$ is a direct sum of cyclic groups since $F / R$ inherits from $F$ the property of being a commutative ring generated by idempotents. In order to show that $G$ is free and to complete the proof of the Theorem, it suffices to show that $G$ is torsion free. Since $F^{+}$is torsion free, it follows at once that $G\left(=F^{+} / R^{+}\right)$is torsion free if and only if $R^{+}$is pure in $F^{+}$. We now appeal to Theorem 1 in [2]. Since $R$ is generated, as a ring, by the idempotents $(x \vee y)+(x \wedge y)-x-y$ in $F, R^{+}$must be pure in $F^{+}$according to Theorem 1 , and the conclusion follows.

The following Corollary of the preceding Theorem settles a question of E. Weinberg posed in [5] and again in [3, p. 368] by J. Rotman; the motivation for the question is explained in [5].

Corollary. The abelian group based on a distributive lattice with relations $a \vee b+a \wedge b=a+b$ is torsion free.

The referee has kindly contributed the following improvement of the preceding result and has brought our attention to some recent related work of G.-C. Rota [6]. We express our gratitude to the referee for these contributions.

If $D L, B R$ and $C R$ denote the categories of distributive lattices, Boolean rings and commutative rings, respectively, we have the functors

$$
C R \stackrel{U_{1}}{\longrightarrow} B R \stackrel{U_{2}}{\longrightarrow} D L,
$$

where $U_{1}$ associates to a commutative ring its Boolean ring of idempotents and $U_{2}$ transforms a Boolean ring into a distributive lattice in the usual way. The functors $U_{1}$ and $U_{2}$ have left adjoints $F_{1}$ and $F_{2}$, respectively: $F_{2}$ adjoins zero and relative complements, and $F_{1}$ is discussed in $\$ 2$ of [1]. Therefore, the composite $U_{3}=U_{2} U_{1}$ has a left adjoint, $F_{3}$, associating to a distributive lattice $L$ the commutative ring $C$ with generators $[x], x \in$ $L$, and relations 


$$
[x \wedge y]=[x][y], \quad[x \vee y]=x+y-x y \text {. }
$$

This is precisely the ring $F / R$ described above. Since $F_{1}$ has free additive structures for its values [1] and since $F_{3}=F_{1} F_{2}$, the same must be true of $F_{3}$.

\section{REFERENCES}

1. G. M. Bergman, Boolean rings of projection maps, J. London Math. Soc. (2) 4 (1972), 593-598. MR 47 \#93.

2. P. Hill, The additive group of commutative rings generated by idempotents, Proc. Amer. Math. Soc. 38 (1973), 499-502. MR 47 \#4986.

3. J. M. Irwin and E. A. Walker (editors), Topics in abelian groups (Proc. Sympos., New Mexico State Univ., 1962), Scott, Foresman, Chicago, Ill., 1963. MR 29 \#5888.

4. G. Nöbeling, Verallgemeinerung eines Satzes von Herrn E. Specker, Invent. Math. 6 (1968), 41-55. MR 38 \#233.

5. E. Weinberg, Free lattice-ordered abelian groups, Math. Ann. 151 (1963), 187-199. MR 27 \#3720.

6. G.-C. Rota, The valuation ring of a distributive lattice, Proc. Houston Lattice Theory Conference (to appear).

DEP ARTMENT OF MATHEMATICS, FLORIDA ST ATE UNIVERSITY, TALLAHASSEE, FLORIDA 32306

DEP ARTMENT OF MATHEM ATICS, ST ATE UNIVERSITY OF NEW YORK AT BUFFALO, BUFF ALO, NEW YORK 14222 